\title{
Underground flux of atmospheric muons and its variations with 25 years of data of the LVD experiment
}

\author{
C. Vigorito* on behalf of the LVD Collaboration \\ Università \& INFN Torino, Italy \\ E-mail: vigoritodto.infn.it \\ G. Bruno, W. Fulgione, A.Molinario \\ INFN Laboratori Nazionali del Gran Sasso, Italy \\ P. Ghia \\ CNRS-IN2P3 Paris, France
}

M. Selvi

INFN Bologna, Italy

\section{G. Trinchero}

INFN Torino, Italy

The Large Volume Detector (LVD) is a 1 kton liquid scintillator detector located at Laboratori Nazionali del Gran Sasso, Italy, at the average depth of $3600 \mathrm{~m}$ w.e.. The experiment has been continuously taking data since June 1992. The muon dataset collected by LVD over 25 years (from 1992 to 2016) consists of $5.5 \cdot 10^{7}$ events, being the largest ever provided by a single detector underground. The analysis of the global time series shows a modulation of muon flux underground with period of $T=(365.1 \pm 0.2)$ days, an amplitude of $1.5 \%$ and a maximum at the beginning of July. A clear correlation with atmospheric temperature variations is present, with a measured coefficient of $\alpha_{T}=0.93 \pm 0.02$. This is in agreement with the expected value at LVD depth and with the value measured by other experiments in the same underground site.

35th International Cosmic Ray Conference - ICRC2017

10-20 July, 2017

Bexco, Busan, Korea

${ }^{*}$ Speaker. 


\section{Introduction}

The flux of muons detected in underground laboratories is directly related to the production of mesons in the stratosphere by hadronic interactions between cosmic rays and the nuclei of air molecules and to the probability that they decay before interacting. This flux shows time variations which are, at first approximation, seasonal. These variations are related to the air density fluctuations, which affect the fraction of mesons decaying to muons energetic enough to reach the underground detector. This effect has been known and studied for many decades [四].

Various experiments at Laboratori Nazionali del Gran Sasso (LNGS), Italy [] [ []] [团], and in other underground sites [5] were able to measure the muon flux underground and its variations on a time scale of few years. In this work we present the results of the analysis of 25 years [1992-2016] of muon flux monitoring with the Large Volume Detector (LVD) at LNGS, at an average depth of $3600 \mathrm{~m}$ w.e.. This represents the largest dataset ever provided so far for muons underground by a single detector.

\section{Temperature effects on the muon rate}

The variations of the temperature in the stratosphere causes variations in the air density, changing the probability with which the mesons produced by primary cosmic rays could interact or decay to high energy muons. In particular, an increase in the temperature of the stratosphere causes a decrease in air density, thus reducing the chance of meson interaction, which in turn results in a larger fraction decaying to produce high energy muons which can eventually reach underground sites. Deviations from the average muon flux measured underground, $\Delta I_{\mu}(t)=\left(I_{\mu}(t)-I_{\mu}^{0}\right)$, can be correlated to variations of the average atmospheric temperature $\Delta T(X)=\left(T(X)-T^{0}(X)\right)$ at a given altitude $X$. The net effect can be written as:

$$
\Delta I_{\mu}(t)=\int_{0}^{\infty} d X W(X) \cdot \Delta T(X, t)
$$

where the weight $W(X)$ (see [司] for details on calculation) reflects the altitude dependence of the production of mesons in the atmosphere (and their decay into muons that can be observed deep underground) and the integral extends over the full atmospheric depth. Assuming the atmosphere as a stratification of $N$ layers with a continuos distribution of temperature and pressure is possible to define an effective temperature, $T_{e f f}$, obtained from a weighted average over atmospheric depth:

$$
T_{e f f}=\frac{\int_{0}^{\infty} d X T(X) W(X)}{\int_{0}^{\infty} d X W(X)} \simeq \frac{\sum_{n=0}^{N} \Delta X_{n} T\left(X_{n}\right) W\left(X_{n}\right)}{\sum_{n=0}^{N} \Delta X_{n} W\left(X_{n}\right)}
$$

assuming that the temperature profile is measured at discrete atmospheric levels, $X_{n}$. Figure $\square$ shows the average temperature profile for the period [1992-2016] at LNGS site and the corresponding weight $W(X)$ as function of the pressure levels $X_{n}$. Defining the effective temperature coefficient as $\alpha_{T}=\frac{T_{e f f}}{I_{\mu}^{0}} \int_{0}^{\infty} d X W(X)$, the relation between effective temperature and muon intensity variations can be written as:

$$
\frac{\Delta I_{\mu}}{I_{\mu}^{0}}=\alpha_{T} \frac{\Delta T_{e f f}}{T_{e f f}} .
$$




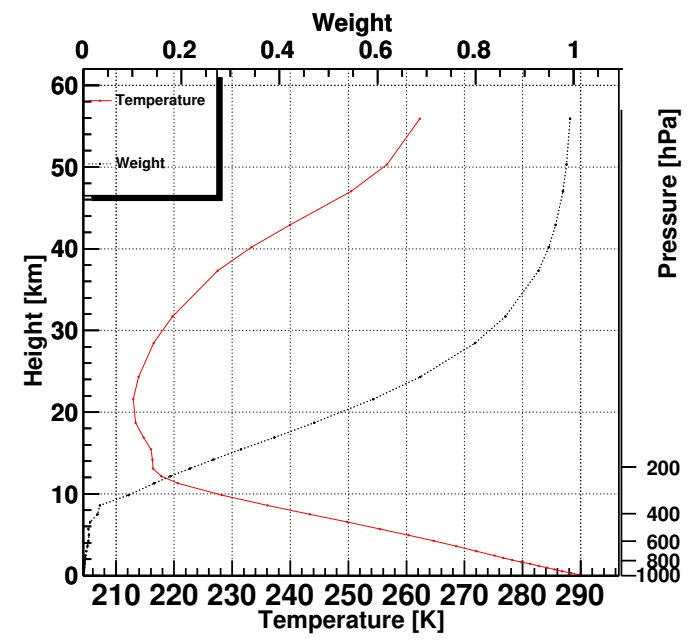

Figure 1: Distribution of the average [19922016] temperature profile at LNGS site and the calculated weight $W(X)$ at different pressure/height levels.

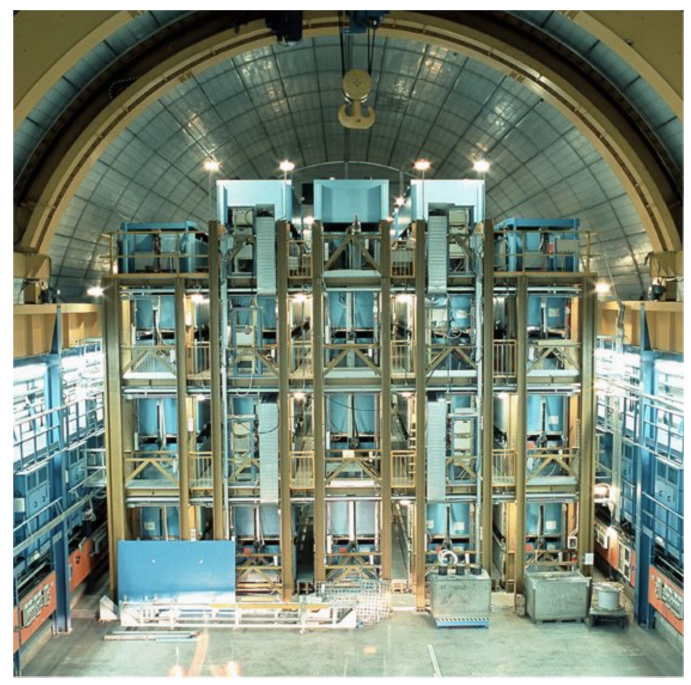

Figure 2: Front view of the LVD experiment in the hall A of the Laboratori Nazionali del Gran Sasso (Italy).

\section{The LVD detector}

LVD (see figure $\mathbb{~ D ) ~ i s ~ a ~} 1000 \mathrm{t}$ liquid scintillator experiment aimed at detecting neutrinos of astrophysical origin. It consists of an array of 840 scintillator self-triggering counters, $1.5 \mathrm{~m}^{3}$ each, viewed from the top by three photomultipliers (PMTs). The counters are organized in a modular and compact geometry which allows to achieve a very high duty cycle. This feature is essential for the search of unpredictable sporadic events like neutrino bursts from Gravitational Stellar Collapses, and it is valuable for the study of time variations of the muon flux underground. LVD has been in operation since 9 June 1992 after a short commissioning phase, its mass increasing from $300 \mathrm{t}$ to its final one, $1000 \mathrm{t}$, in January 2001. The LVD active mass and duty cycle evolution in time, updated to May 2017, are shown in figure B. The LVD trigger logic (extensively described in [四]) is based on the 3-fold coincidence of the PMTs in a single counter corresponding to an energy threshold $E_{t h} \sim 4 \mathrm{MeV}$. The energy resolution of the counter is $\Delta E / E \sim 15 \%$ at $10 \mathrm{MeV}$. The time stamp of triggered events has a relative time precision of $12.5 \mathrm{~ns}$ with an absolute accuracy of 100 ns.

\section{Temperature Data}

The temperature data has been obtained from the European Center for Medium-range Weather Forecasts (ECMWF ERA-Interim data) [8]. It exploits different type of observations (e.g. surface, satellite, and upper air sounding) at many locations, and then uses a global atmospheric model to interpolate them to a particular location. We considered in this analysis the precise coordinates of the LNGS underground halls: $13.5333^{\circ} \mathrm{E}, 42.4275^{\circ} \mathrm{N}$. The model provides atmospheric temperatures at 37 discrete pressure levels in the [1-1000] hPa range, four times a day at $00.00 \mathrm{~h}, 06.00 \mathrm{~h}$, 

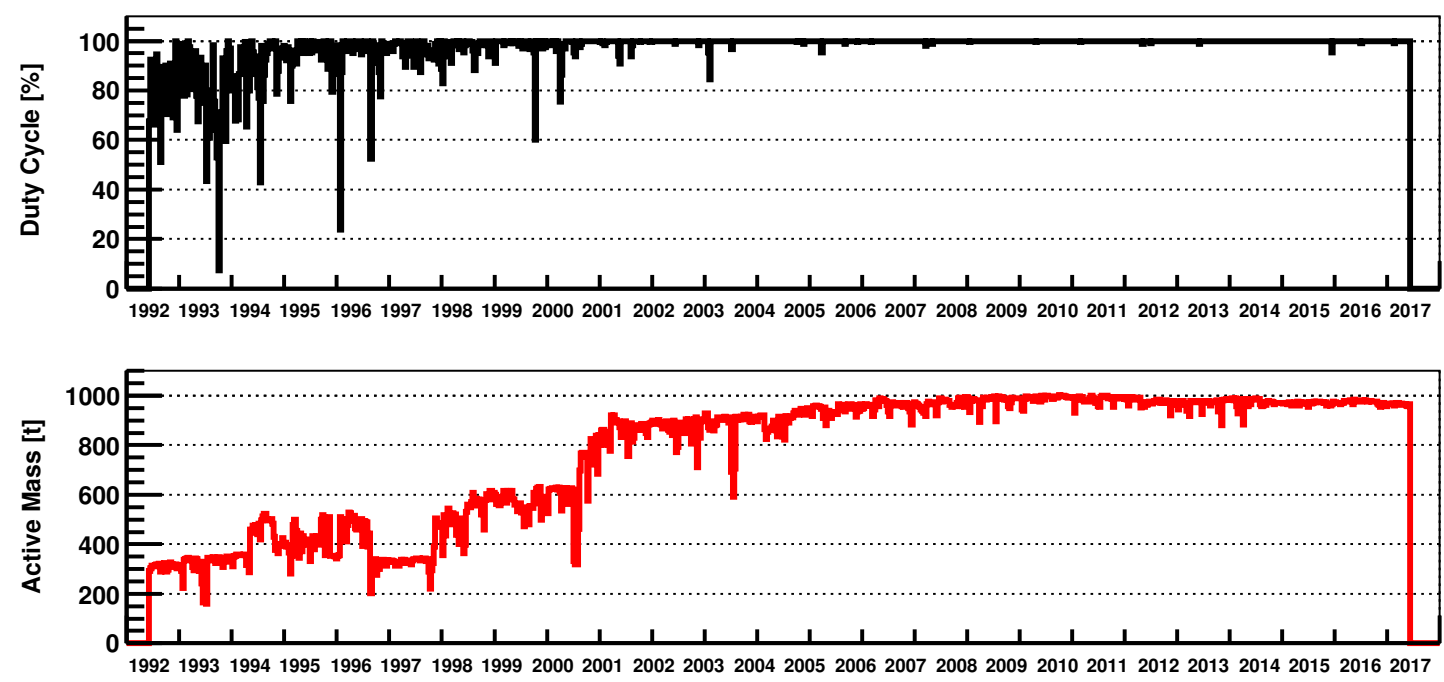

Figure 3: LVD duty cycle and active mass as a function of time from 1992, June $9^{\text {th }} 1992$ to 2017, May $28^{\text {th }}$.

$12.00 \mathrm{~h}$, and $18.00 \mathrm{~h}$. Based on this data set, the $T_{\text {eff }}$ is calculated following equation 2.2 four times a day providing a mean daily value. The variance of the four daily values is assumed as estimate of the uncertainty of the mean.

The daily value of $T_{\text {eff }}$, over the period considered in the present analysis, is shown in figure 目: a simple average gives $\left\langle T_{\text {eff }}^{o}\right\rangle=(220.290 \pm 0.006) \mathrm{K}$.

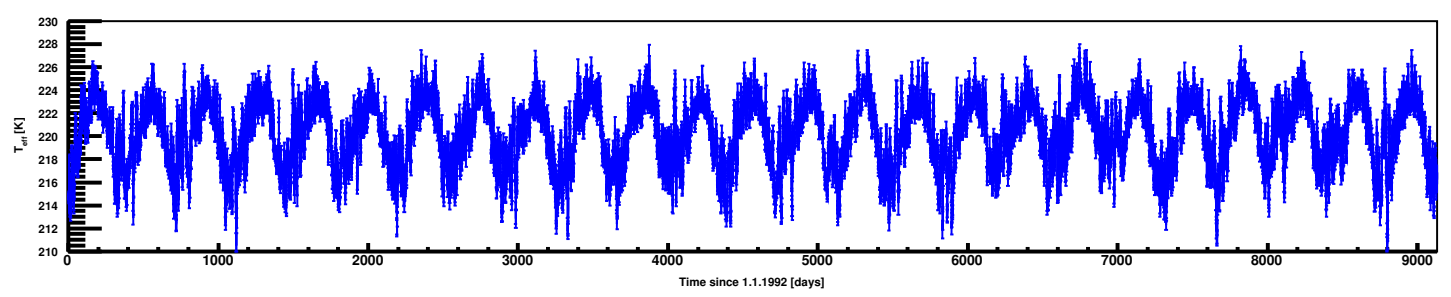

Figure 4: Daily values of $T_{e f f}$ from 1992 up to 2016 as obtained by using ECMWF Era-interim data.

\section{LVD Muon Data}

Muons are identified in LVD through the time coincidence of signals with an energy release $E \geq 10 \mathrm{MeV}$ in two or more counters within a time window of $175 \mathrm{~ns}$, which is large enough to include the time jitter of the PMT's transit time. Data selection is performed via the standard quality cuts that have been defined in the search for neutrino burst [Q]. Additionaly, the muon-like events associated to the CNGS (CERN Neutrino to Gran Sasso [ए]]) neutrino beam in the period [2006-2012] have been excluded if in coincidence with the time of the beam spills inside a time window of $[-10:+20.5] \mu \mathrm{s}$, being $10.5 \mu \mathrm{s}$ the spill duration. The resulting dataset consists of $5.5 \cdot 10^{7}$ muons for a total livetime of 8403 days. The measured daily muon rate is shown in figure 
5 and it is mainly affected by the different detector configurations over the time, as shown in figure 3]. The muon flux underground $I_{\mu}(t)$ is then obtained through the ratio between the measured daily

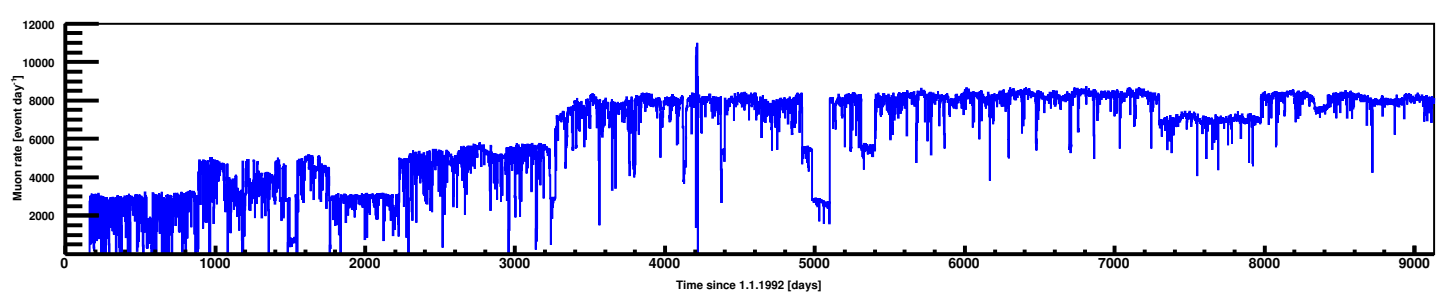

Figure 5: Daily muon rate in LVD (1 day binning) over the full data set.

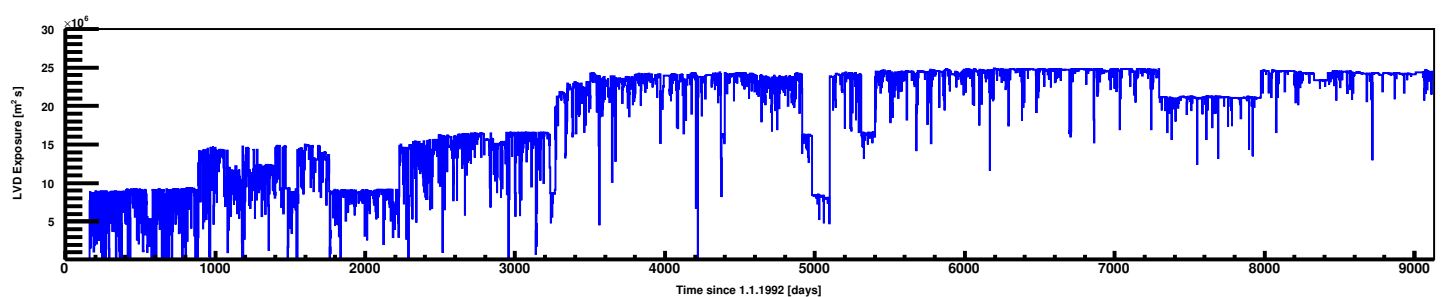

Figure 6: Daily LVD exposure, the geometrical acceptance times the daily livetime (1 day binning).

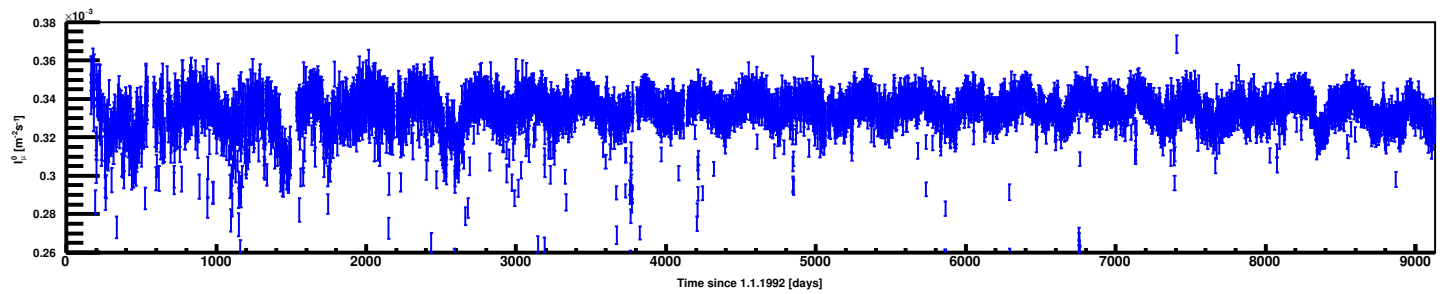

Figure 7: Daily values of muon flux underground $I_{\mu}(t)$ from 1992 up to 2016: only statistical error bars are shown. The bigger fluctuations, observed till the beginning of 2001 (day 3288), are due to the lower active mass of the detector during construction.

muon rate (fig. (1) and the simulated detector exposure (see fig. 6 , i.e the geometrical acceptance times the livetime), which changes according to the LVD active mass configurations and duty cycle as shnown in figure B]. A detailed description of the simulation can be found here [ए]]. For the full detector configuration (i.e. $M=1000 \mathrm{t}$ ) the geometrical acceptance, averaged over the cosmic muon arrival directions in the hall A of LNGS, corresponds to $S=(298 \pm 3) \mathrm{m}^{2}$, where the uncertainty $(1 \%)$ is mainly dominated by the systematic errors assumed in the muon direction. The obtained daily detector exposure is shown in figure $\mathbf{6}$, and the corresponding muon flux is shown in figure $\square$. The bigger fluctuations which are observed till the beginning of 2001 (day 3288) are due to the lower active mass of the detector during construction phase. The average value of the muon flux is $I_{\mu}^{0}=(3.3332 \pm 0.0005) \cdot 10^{-4} \mathrm{~m}^{-2} \mathrm{~s}^{-1}$, where only statistical errors have been considered. A $1 \%$ effect should be added to include systematic errors in the geometrical acceptance $S$ calculation. 


\section{Results}

The daily muon flux of figure $\square$ has been fitted with a sinusoidal function

$$
I_{\mu}(t)=I_{\mu}^{0}+\Delta I_{\mu} \cos \left(\frac{2 \pi}{T}(t-\phi)\right)
$$

obtaining a period of $T=(365.1 \pm 0.2) \mathrm{d}$, which is compatible with what expected from the yearly temperature modulation. The amplitude $\Delta I_{\mu}$ and the phase $\phi$ of the modulation can be better evaluated by projecting and averaging the 25 years dataset into one single year, as shown in figure 8 . We assume that there is not a long term modulation lying under the first armonic and we neglect the short time variations. We use again the fit function in $6 . \mathbb{W}$, but fixing $T=365 \mathrm{~d}$, and including systematic errors of muon flux values. We obtain $\Delta I_{\mu}=(5.2 \pm 0.3) \cdot 10^{-6} \mathrm{~m}^{-2} \mathrm{~s}^{-1}(\sim 1.5 \%)$ and a phase $\phi=(187 \pm 3) \mathrm{d}$ (corresponding to the beginning of July).

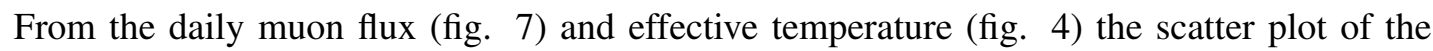
relative daily variations values $\frac{\Delta I_{\mu}}{I_{\mu}^{0}}$ and $\frac{\Delta T_{\text {eff }}}{T_{\text {eff }}^{0}}$ is shown in figure $\boldsymbol{Q}$. A clear correlation between the two distributions is obtained.

It is possible to extract from here the value of $\alpha_{T}$ (equation 2.3.3) performing a linear regression which accounts for error bars. We get a value of $\alpha_{T}=0.93 \pm 0.02$ (correlation coefficient $r=$ 0.49 ), which is in agreement with the expected value of $\alpha_{T, L N G S}=0.92 \pm 0.02$ at LNGS depth [[]] [G]. This result confirms the values that were measured by others detectors at LNGS [], [[]], [G]. Figure 10 shows the measured values of $\alpha_{T}$ by various experiments at different depths, together with the theoretical prediction including muon production by pions and kaons.

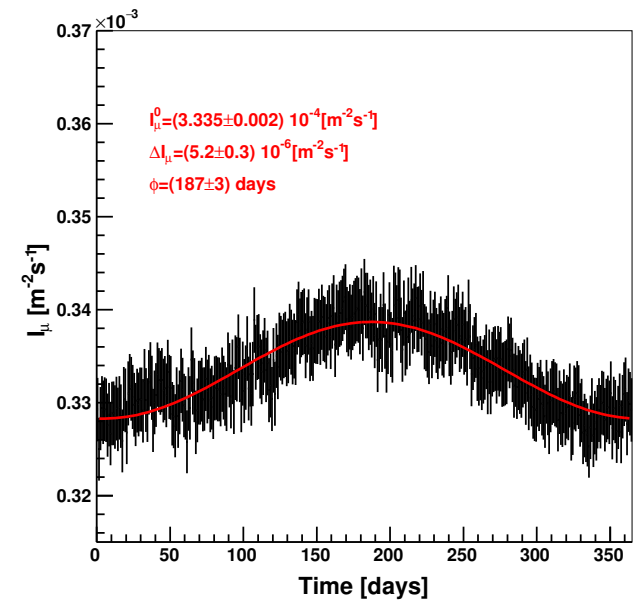

Figure 8: Muon flux underground with 25 years dataset folded onto one year period. The vertical bars account for both statistical and systematic errors. The red line is the sinusoidal fit according to formula 6.$]$ with $T=365 \mathrm{~d}$.

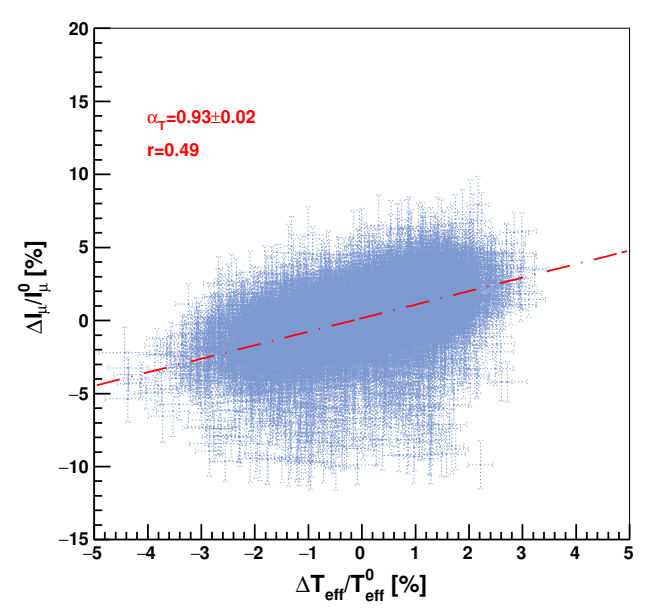

Figure 9: Scatter plot of daily values of $\frac{\Delta I_{\mu}}{I_{\mu}^{0}}$ and $\frac{\Delta T_{\text {eff }}}{T_{\text {eff }}^{0}}$ : the dashed red line corresponds to the linear regression of the whole data set. 


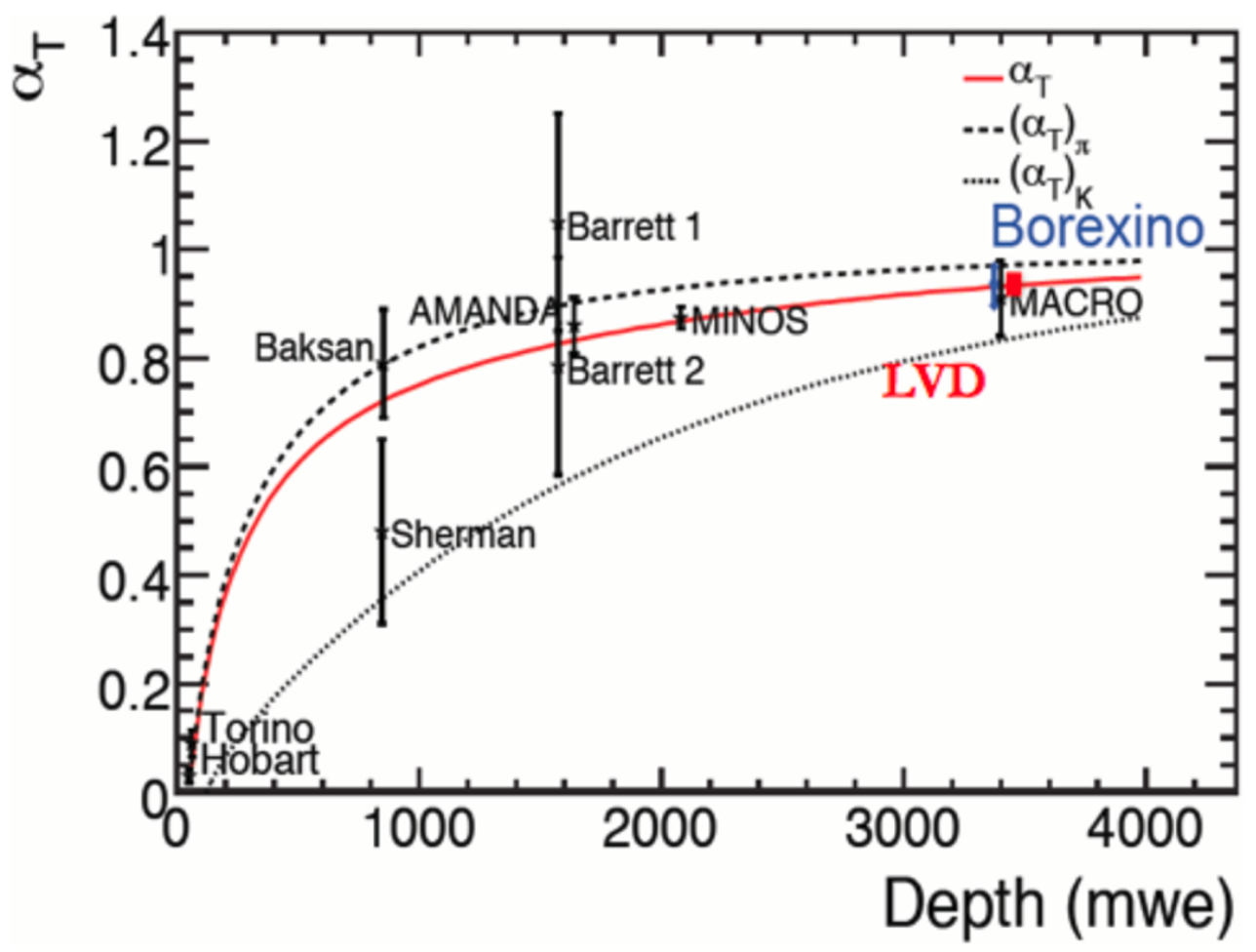

Figure 10: Measured values for the effective temperature coefficient $\alpha_{T}$ at different site depths. The results of this analysis (in red) compared with those of other detectors. The red line is the predicted value including both muon production by pions and kaons, shown separately by the dashed lines. See [5] for details.

\section{Conclusions}

We have analized $5.5 \times 10^{7}$ muon events detected by the LVD experiment over 8403 live days in the [1992-2016] period to search for variations of the muon flux underground in correlation with the atmospheric effective temperature variations at LNGS site.

The average value of the muon flux over 25 years of data is:

$$
I_{\mu}^{0}=(3.3335 \pm 0.002[\text { stat. }] \pm 0.03[\text { sys }]) 10^{-4} \mathrm{~m}^{-2} \mathrm{~s}^{-1}
$$

being the error dominated by the sistematic uncertainty on the geometrical acceptance correction (1\%). A clear modulation with a time period $T=1 \mathrm{y}$ is observed with an amplitude of $1.5 \%$ and a phase corresponding to beginning of July. Relative variations of muon flux are correlated to the effective temperature relative variations with a coefficient $\alpha_{T}=(0.93 \pm 0.02)$, which is well in agreement with previous measurements at LNGS site and also with the expected value for the LNGS depth.

A full paper on this analysis is in preparation and expected to be published later in 2017. 


\section{References}

[1] P.H. Barrett et al., Rev. Mod. Phys. 24 (1952) 133.

[2] M. Ambrosio et al. (MACRO coll.), Astropart. Phys. 7 (1997) 109.

[3] G. Bellini et al., J. of Cosmol. and Astropart. Phys. 5 (2012) 15.

[4] M. Agostini et al., Astropart.Phys. 84 (2016) 29-35.

[5] P. Adamson et al., Phys. Rev. D 81 (2010) 012001.

[6] E. W. Grashorn et al., Astropart. Phys. 33 (2010) 140.

[7] N. Yu. Agafonova et al. (LVD coll.), Astropart. Phys. 27 (2007) 254-270.

[8] ECMWF, European Centre for Medium-Range Weather Forecast, http://www.ecmwf.int

[9] N. Yu. Agafonova et al. (LVD coll.), Astrophys. J. 802 (2015), no. 1, 47.

[10] G. Acquistapace et al., CERN-98-02, INFN/AE-98/05; CERN-Sl/99-034(DI), INFN/AE-99/05 Addendum (1998).

[11] M. Selvi (for the LVD coll.), in proc. of the 31st ICRC (2009). 